\title{
Resistance Distances in Linear Polyacene Graphs
}

\author{
Dayong Wang ${ }^{1}$ and Yujun Yang $^{2 *}$ \\ ${ }^{1}$ Business School, Hohai University, Nanjing, China, ${ }^{2}$ School of Mathematics and Information Sciences, Yantai University, Yantai, \\ China
}

The resistance distance between any two vertices of a connected graph is defined as the net effective resistance between them in the electrical network constructed from the graph by replacing each edge with a unit resistor. In this article, using electric network approach and combinatorial approach, we derive exact expression for resistance distances between any two vertices of polyacene graphs.

Keywords: hexagonal lattice, local rules, polyacene graph, resistance distance, circuit reduction

\section{INTRODUCTION}

Let $G=(V(G), E(G))$ be a connected graph. It is interesting to consider distance functions on $G$. The most natural and best-known distance function is the shortest path distance. For any two vertices $i, j \in V(G)$, the shortest path distance between $i$ and $j$, denoted by $d_{G}(i, j)$, is defined as the length of a shortest path connecting $i$ and $j$. Two decays ago, another novel distance function, named resistance distance, was identified by Klein and Randić [1]. The concept of resistance distance originates from electrical circuit theory. If we view $G$ as an electrical network $N$ by replacing each edge of $G$ with a unit resistor, then the resistance distance [1] between $i$ and $j$, denoted by $\Omega_{G}(i, j)$, is defined as the net effective resistance between the corresponding nodes in the electrical network $N$. In contrast to the shortest path distance, the resistance distance has a notable feature that if $i$ and $j$ are connected by more than one path, then they are closer than they are connected by the only shortest path. So it is suggested that resistance distance is more appropriate to deal with wave-like motion in the network, like the communication in chemical molecules. In addition, it turns out that the resistance distance has some pure mathematical interpretations, which could be expressed in terms of the generalized inverse of the Laplacian matrix [1], the number of spanning trees and spanning bi-trees [2], and random walks on graphs $[3,4]$.

Besides being an intrinsic graph metric and an important component of electrical circuit theory, resistance distance also turns out to have important applications in chemistry. For this reason, resistance distance has been widely studied in the mathematical, chemical, and physical literature. In the study of resistance distance, the main focus is placed on the problem of computation of resistance distance. This problem has been a classical problem in electrical network theory studied by numerous researchers for a long time. Besides, it is also relevant to a wide range of problems ranging from random walks, the theory of harmonic functions, to lattice Green's functions. Consequently, this problem has attracted much attention, and many researchers have devoted themselves to it. Up to now, resistance distances have been computed for many interesting (classes of) graphs, with emphasis being placed on some highly concerned electrical networks and chemical interesting graphs. For example, resistance distances have been computed for Platonic solids [5], and for some fullerene graphs including buckminsterfullerene [6], circulant graphs [7], distance-regular graphs [8, 9], pseudo-distance-regular graphs [10], wheels and fans [11], Cayley graphs over finite abelian groups [12], complete graph minus $N$ edges [13], resistor network embedded on a globe [14], Möbius ladder [15], $m \times n$ cobweb network [16], complete $n$-partite graphs [17], $m \times n$ resistor network [18],

Wang D and Yang Y (2021) Resistance Distances in Linear Polyacene Graphs. 
ladder graph [19], n-step network [20], Cayley graphs on symmetric groups [21], Apollonian network [22], Sierpinski Gasket Network [23], generalized decorated square and simple cubic network lattices [24], self-similar $(x, y)$-flower networks [25], almost complete bipartite graphs [26], straight linear 2-trees [27], and path networks [28].

It is interesting to note that a good deal of attention has been paid on resistance distances in plane networks, such as Platonic solids, fullerene graphs, wheels, fans, ladder graphs, Apollonian network, Sierpinski Gasket Network, $m \times n$ resistor network, and straight linear 2-tree. Motivated by this fact, we are devoted to considering other interesting plane networks. In this article, we take the linear polyacene graphs into consideration. It is well known that the linear polyacene graphs are graph representations of an important class of benzenoid hydrocarbons, and it is an interesting class of plane hexagonal networks. We use $L_{n}$ to denote the linear polyacene graph with $n-1$ benzenoid rings (i.e., hexagons), as shown in Figure 1. Using electrical network approach and resistance distance local rules, we derive exact expression for resistance distances between any two vertices of $L_{n}$.

\section{RESISTANCE DISTANCES IN LINEAR POLYACENE GRAPHS}

Let $L_{n}$ be the linear polyacene graph with $n-1$ benzenoid rings. Obviously, $L_{n}$ has $4 n-2$ vertices and $5 n-4$ edges. For convenience, we label the vertices in $L_{n}$ as in Figure 1. We partite the vertex set of $L_{n}$ into two classes: $V_{1}=$ $\left\{p_{1}, p_{2}, \ldots, p_{n}, q_{1}, q_{2}, \ldots, q_{n}\right\} \quad$ and $\quad V_{2}=\left\{s_{1}, s_{2}, \ldots, s_{n-1}\right.$, $\left.t_{1}, t_{2}, \ldots, t_{n-1}\right\}$. To compute resistance distances between any two vertices of $L_{n}$, we take two steps. In the first step, we compute resistance distances between vertices in $V_{1}$. To this end, we first view $L_{n}$ as a weighted ladder graph $L_{n}^{*}$ by simply replacing all the paths $p_{i} s_{i} p_{i+1}$ and $q_{i} t_{i} q_{i+1}(1 \leq i \leq n-1)$ by edges of resistance 2 . Then, by making use of the electric network approach as inspired in [19], we obtain resistance distances between vertices in $V_{1}$. Next, for the second step, using the results obtained in the first step together with resistance distance local rules, we derive expressions for resistance distances between the remaining pairs of vertices.

Before stating the main result, we introduce the elegant resistance distance local rules, which will be frequently used later. For any vertex $a \in V(G)$, we use $n_{G}(a)$ to denote the set of neighbors of $a$. Then, we have the following sum rules for resistance distances.

Lemma 2.1 [29]. Let $G=(V(G), E(G))$ be a connected graph with $n(n \geq 2)$ vertices. Then,

1) For any $a, b \in V(G)(a \neq b)(a \neq b)$

$$
\Delta_{a} \Omega_{G}(a, b)+\sum_{i \in n_{G}(a)}\left(\Omega_{G}(i, a)-\Omega_{G}(i, b)\right)=2,
$$

where $\Delta_{a}$ denotes the degree of the vertex a.

2) For any three different vertices $a, b, c \in V$,

$$
\Delta_{c}\left(\Omega_{G}(c, a)-\Omega_{G}(c, b)\right)+\sum_{i \in n_{G}(c)}\left(\Omega_{G}(i, b)-\Omega_{G}(i, a)\right)=0 .
$$

Now, we are ready for the main theorem. For simplicity, we let $\alpha=3-2 \sqrt{2}$, and define $f(x, y)$ and $g(x, y)$ as follows:

$$
\begin{aligned}
& f(x, y)=\left(1-\alpha^{x-y}\right)\left(2-\alpha^{x+y-1}+\alpha^{2 y-1}+\alpha^{2 n-2 x+1}\left(1-\alpha^{x-y}-2 \alpha^{x+y-1}\right)\right), \\
& g(x, y)=\left(1+\alpha^{x-y}\right)\left(2+\alpha^{x+y-1}+\alpha^{2 y-1}+\alpha^{2 n-2 x+1}\left(1+\alpha^{x-y}+2 \alpha^{x+y-1}\right)\right) .
\end{aligned}
$$

Then, the main result is given in the following.

Theorem 2.2. The resistance distances between any two vertices in the linear polyacene graph $L_{n}$ can be computed as follows.

$$
\begin{gathered}
\Omega_{L_{n}}\left(p_{i}, p_{j}\right)=i-j+\frac{f(i, j)}{4 \sqrt{2}\left(1-\alpha^{2 n}\right)}, \\
\Omega_{L_{n}}\left(q_{i}, p_{j}\right)=i-j+\frac{g(i, j)}{4 \sqrt{2}\left(1-\alpha^{2 n}\right)}, \\
\Omega_{L_{n}}\left(s_{i}, p_{j}\right)=i-j+\frac{3}{4}-\frac{f(i+1, i)}{16 \sqrt{2}\left(1-\alpha^{2 n}\right)}+\frac{f(i, j)+f(i+1, j)}{8 \sqrt{2}\left(1-\alpha^{2 n}\right)}, \\
\Omega_{L_{n}}\left(s_{i}, q_{j}\right)=j-i-\frac{1}{4}+\frac{f(j+1, j)}{16 \sqrt{2}\left(1-\alpha^{2 n}\right)}+\frac{g(j, i)+g(j, i+1)}{8 \sqrt{2}\left(1-\alpha^{2 n}\right)}, \\
\Omega_{L_{n}\left(s_{i}, s_{j}\right)=\frac{1}{2}-i+j} \\
-\frac{f(i+1, i)+f(j+1, j)+f(j, i)+f(j+1, i)+f(j, i+1)+f(j+1, i+1)}{16 \sqrt{2}\left(1-\alpha^{2 n}\right)},
\end{gathered}
$$

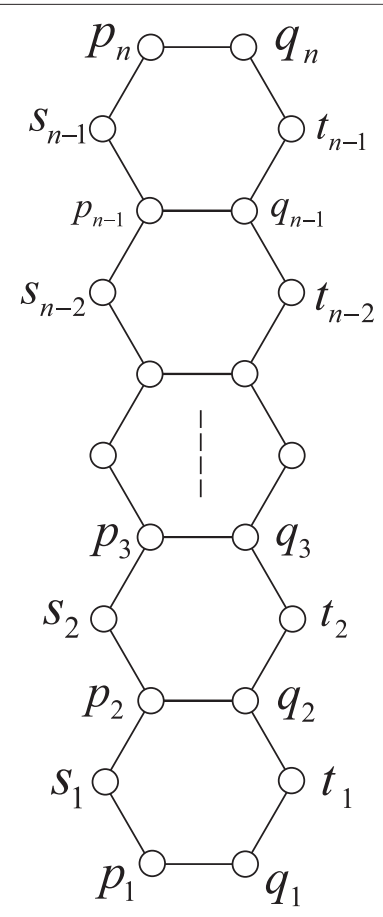

FIGURE 1 | Linear polyacne graph $L_{n}$ and its vertex labeling. 


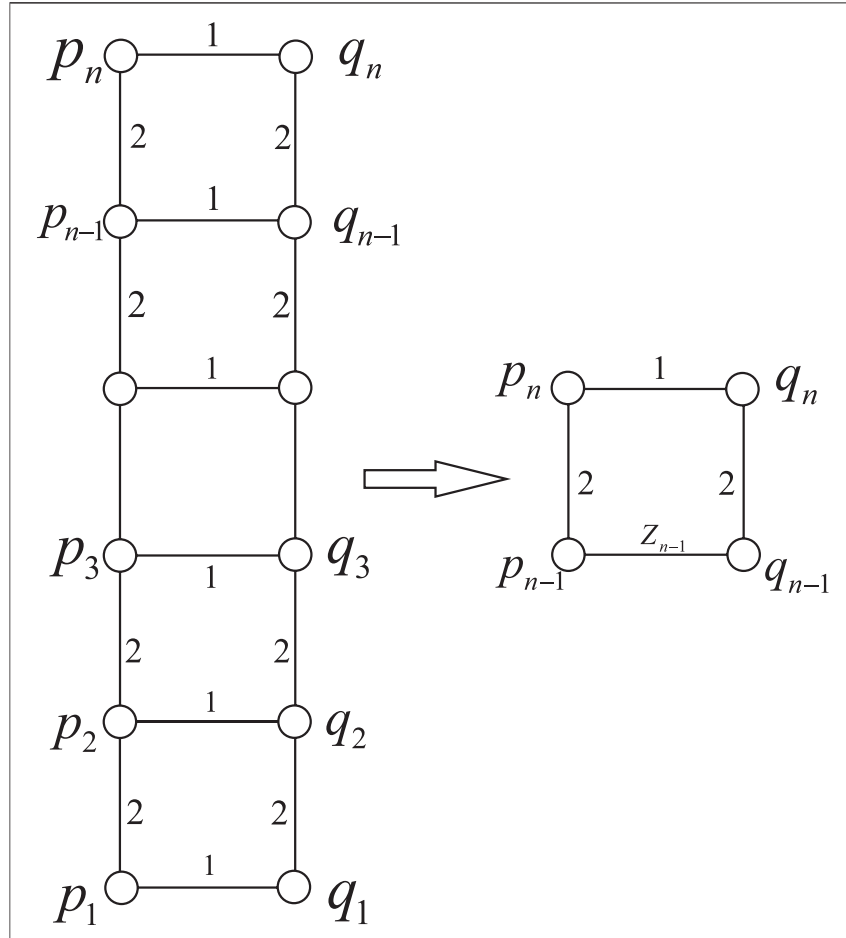

FIGURE 2 | Weighted ladder graph $L_{n}^{*}$ (left) and the circuit reduction of $L_{n-1}^{*}$ with respect to $p_{n-1}$ and $q_{n-1}$ (right).

$$
\begin{aligned}
\Omega_{L_{n}}\left(s_{i}, t_{j}\right)=\frac{1}{2}+i-j \\
+\frac{g(i, j)+g(i, j+1)+g(i+1, j)+g(i+1, j+1)-f(i+1, i)}{16 \sqrt{2}\left(1-\alpha^{2 n}\right)} \\
\quad-\frac{f(i+1, i)+f(i+2, i+1)}{32 \sqrt{2}\left(1-\alpha^{2 n}\right)} .
\end{aligned}
$$

Proof. We divide the proof into two steps.

Step 1. Computation of resistance distances between any two vertices in $V_{1}$.

To compute resistance distances between vertices in $V_{1}$, we view $L_{n}$ as a weighted ladder graph $L_{n}^{*}$ by simply replacing all the paths $p_{i} s_{i} p_{i+1}$ and $q_{i} t_{i} q_{i+1}(1 \leq i \leq n-1)$ by edges of resistance 2 , see Figure 2 (left). Clearly, $\Omega_{L_{n}^{*}}(p, q)=\Omega_{L_{n}}(p, q)$ holds for all $p, q \in V\left(L_{n}^{*}\right)$.

First, we compute resistance distances between the end vertices $p_{1}, p_{n}, q_{1}$, and $q_{n}$. let $x_{n}:=\Omega_{L_{n}^{*}}\left(p_{n}, p_{1}\right)$, $y_{n}:=\Omega_{L_{n}^{*}}\left(p_{n}, q_{1}\right)$, and $z_{n}:=\Omega_{L_{n}^{*}}\left(p_{n}, q_{n}\right)$. Clearly, $L_{n}^{*^{n}}$ can be obtained from $L_{n-1}^{*}$ by adding two vertices $p_{n}$ and $q_{n}$, and the three edges with end vertices $\left\{p_{n-1}, p_{n}\right\},\left\{p_{n}, q_{n}\right\}$, and $\left\{q_{n}, q_{n-1}\right\}$, as shown in Figure 2 (right). Hence, according to rules for series and parallel circuits, $z_{n}$ could be expressed in term of $z_{n-1}$ as

$$
z_{n}=\frac{z_{n-1}+4}{z_{n-1}+5}, \quad \forall n \geq 2
$$

with initial condition $z_{1}=1$. Solving the recurrence relation by Mathematica [30], we obtain

$$
z_{n}=-2(1+\sqrt{2})+\frac{4 \sqrt{2}}{1-(3-2 \sqrt{2})^{2 n}}, \quad n \geq 1 .
$$

Specially, we have $z_{1}=1, z_{2}=\frac{5}{6}, z_{3}=\frac{29}{35}$, and $z_{4}=\frac{169}{204}$. It is easily checked that $z_{n}$ can also be expressed as

$$
z_{n}=-2(1+\sqrt{2})+\frac{4 \sqrt{2}(3+2 \sqrt{2})^{n}}{(3+2 \sqrt{2})^{n}-(3-2 \sqrt{2})^{n}}, \quad n \geq 1 .
$$

We proceed to use $z_{n}$ to find explicit formulas for $x_{n}$ and $y_{n}$. To this end, we make circuit reduction to the subgraph $L_{n}^{*}$ of $L_{n+1}^{*}$ with respect to $p_{n}, q_{n}$, and $p_{1}$, where $n \geq 1$. Precisely speaking, we reduce $L_{n}^{*}$ to a $Y$-shaped graph which has outer vertices $p_{n}, q_{n}$, and $p_{1}$. We use $A, B$, and $C$ to denote the effective resistances between end vertices of those edges of the $Y$-shaped graph. Then, we have $B+C=y_{n}, A+C=x_{n}$, and $A+B=z_{n}$. Solving these equations, we get

$$
A=\frac{x_{n}-y_{n}+z_{n}}{2}, B=\frac{-x_{n}+y_{n}+z_{n}}{2}, C=\frac{x_{n}+y_{n}-z_{n}}{2} \text {. }
$$

On the other hand, by parallel and series connection rules, we have $x_{n+1}=\frac{(A+2)(B+3)}{z_{n}+5}+C$ and $y_{n+1}=\frac{(B+2)(A+3)}{z_{n}+5}+C$. So, it follows that

$$
\begin{aligned}
x_{n+1}= & \frac{\left(x_{n}-y_{n}+z_{n}+4\right)\left(-x_{n}+y_{n}+z_{n}+6\right)}{4\left(z_{n}+5\right)} \\
& +\frac{x_{n}+y_{n}-z_{n}}{2}, \quad n \geq 1, \\
y_{n+1}= & \frac{\left(-x_{n}+y_{n}+z_{n}+4\right)\left(x_{n}-y_{n}+z_{n}+6\right)}{4\left(z_{n}+5\right)} \\
& +\frac{x_{n}+y_{n}-z_{n}}{2}, \quad n \geq 1,
\end{aligned}
$$

with initial conditions $x_{1}=0$ and $y_{1}=1$. Eq. 2.10 minus Eq. 2.11 yields

$$
x_{n+1}-y_{n+1}=\frac{x_{n}-y_{n}}{z_{n}+5} .
$$

Set $t_{n}:=x_{n}-y_{n}$. It follows that

$$
t_{n+1}=\frac{t_{n}}{z_{n}+5}, \quad n \geq 1 \text { and } t_{1}=-1
$$

Thus, we have

$$
t_{n+1}=-\prod_{k=1}^{n} \frac{1}{z_{k}+5}
$$

Since $\frac{1}{z_{k}+5}=\frac{(3+2 \sqrt{2})^{k}-(3-2 \sqrt{2})^{k}}{(3+2 \sqrt{2})^{k+1}-(3-2 \sqrt{2})^{k+1}}$, using Eq. 2.9 and doing some algebraic calculations, we get

$$
t_{n}=\frac{-4 \sqrt{2}}{(3+2 \sqrt{2})^{n}-(3-2 \sqrt{2})^{n}}, \quad n \geq 1 .
$$

This could also be rewritten as $t_{n}=\frac{-4 \sqrt{2}(3-2 \sqrt{2})^{n}}{1-(3-2 \sqrt{2})^{2 n}}$, for all $n \geq 1$. Now, we come back to solve $x_{n}$ and $y_{n}$. By using $x_{n}=t_{n}+y_{n}$, Eqs 2.8-2.14 and doing some algebra, Eq. 2.11 becomes 
$y_{n+1}=y_{n}+\frac{2 \sqrt{2}}{1-(3-2 \sqrt{2})^{n+1}}-\frac{2 \sqrt{2}}{1-(3-2 \sqrt{2})^{n}}+1, \quad n \geq 1$ and $y_{1}=1$.

Solving the recursion relation, we get

$$
y_{n}=n-2-\sqrt{2}+\frac{2 \sqrt{2}}{1-(3-2 \sqrt{2})^{n}}, \quad n \geq 1 .
$$

Now, by Eqs 2.14-2.16, together with the relation $x_{n}=t_{n}+y_{n}$, we get

$$
x_{n}=n-2-\sqrt{2}+\frac{2 \sqrt{2}}{1+(3-2 \sqrt{2})^{n}}, \quad n \geq 1 .
$$

Next, we proceed to compute $\Omega_{L_{n}^{*}}\left(p_{n}, p_{i}\right), \Omega_{L_{n}^{*}}\left(p_{n}, q_{i}\right)$, and $\Omega_{L_{n}^{*}}\left(p_{i}, q_{i}\right)$, where $n>i>1$. To achieve our goal, we consider $L_{n}^{*}$ as the union of three graphs: the upper part of $p_{i+1}$ and $q_{i+1}$, the lower part of $p_{i}$ and $q_{i}$, and the middle part consisting of $p_{i+1}, q_{i+1}$, $p_{i}$, and $q_{i}$, as shown in Figure 3. Note that the upper and the lower graphs are corresponding to the graphs $L_{n-i}^{*}$ and $L_{i}^{*}$, respectively. We make circuit reductions as illustrated in Figure 3. First, make the circuit reduction of the upper part with respect to $p_{n}, p_{i+1}$, and $q_{i+1}$ to obtain a $Y$-shaped graph, and assume that resistances along its edges are $M, N$, and $K$. Then, reduce the lower part of $p_{i}$ and $q_{i}$ to be edge with resistance $\Omega_{L_{n}^{*}}\left(p_{i}, q_{i}\right)=z_{i}$. We could find that

$$
M+N=x_{n-i}, M+K=y_{n-i}, N+K=z_{n-i} .
$$

Note that

$$
\begin{aligned}
& x_{n}+y_{n}-z_{n}=2 n-2, \\
& x_{n}-y_{n}+z_{n}=-2-2 \sqrt{2}+\frac{4 \sqrt{2}}{1+(3-2 \sqrt{2})^{n}}, \\
& -x_{n}+y_{n}+z_{n}=-2-2 \sqrt{2}+\frac{4 \sqrt{2}}{1-(3-2 \sqrt{2})^{n}} .
\end{aligned}
$$

Solving $M, N$, and $K$, we obtain

$$
\begin{aligned}
& M=\frac{x_{n-i}+y_{n-i}-z_{n-i}}{2}=n-i-1, \\
& N=\frac{x_{n-i}-y_{n-i}+z_{n-i}}{2}=-1-\sqrt{2}+\frac{2 \sqrt{2}}{1+(3-2 \sqrt{2})^{n-i}}, \\
& K=\frac{-x_{n-i}+y_{n-i}+z_{n-i}}{2}=-1-\sqrt{2}+\frac{2 \sqrt{2}}{1-(3-2 \sqrt{2})^{n-i}} .
\end{aligned}
$$

Then, applying parallel and series connection rules to the reduced circuit in Figure 3, we obtain

$$
\begin{aligned}
& \Omega_{L_{n}^{*}}\left(p_{n}, p_{i}\right)=\frac{(N+2)\left(K+z_{i}+2\right)}{z_{n-i}+z_{i}+4}+M, \\
& \Omega_{L_{n}^{*}}\left(p_{n}, q_{i}\right)=\frac{(K+2)\left(N+z_{i}+2\right)}{z_{n-i}+z_{i}+4}+M, \\
& \Omega_{L_{n}^{*}}\left(p_{i}, q_{i}\right)=\frac{z_{i}\left(z_{n-i}+4\right)}{z_{n-i}+z_{i}+4} .
\end{aligned}
$$

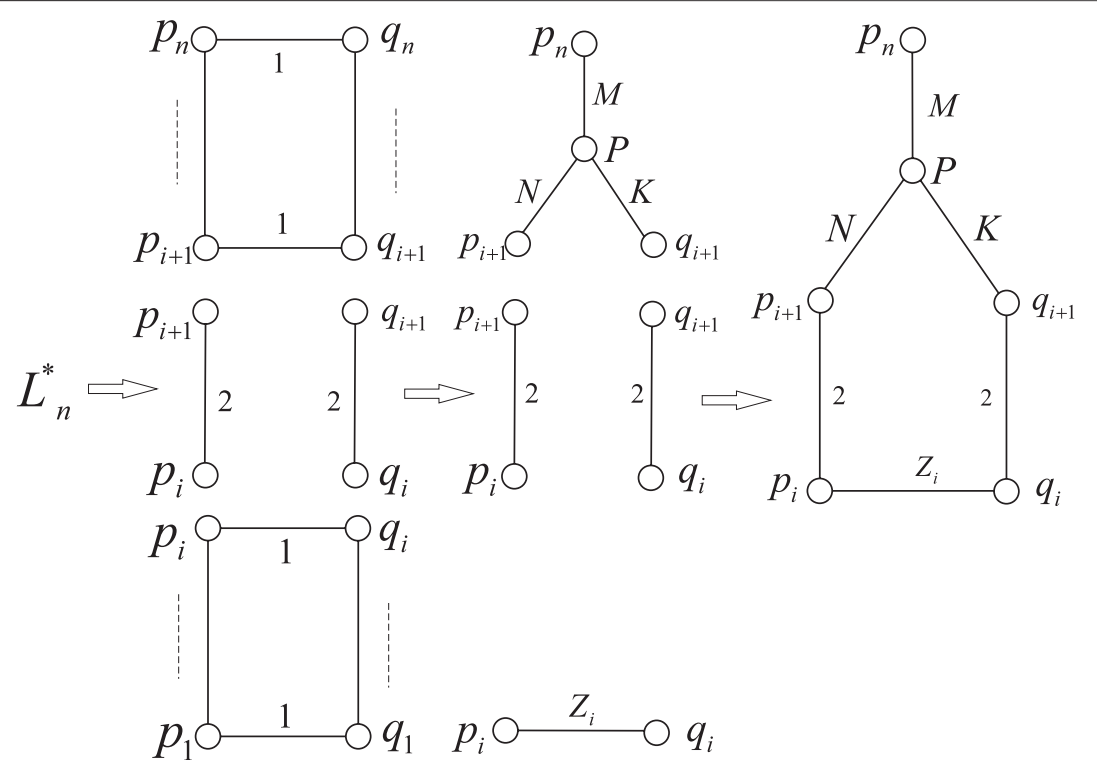

FIGURE $3 \mid L_{n}^{*}$ and circuit reduction to find $\Omega_{L_{n}^{*}}\left(p_{n}, p_{i}\right), \Omega_{L_{n}^{*}}\left(q_{n}, p_{i}\right)$, and $\Omega_{L_{n}^{*}}\left(p_{i}, q_{i}\right)$. 
Substituting Eqs 2.8-2.20 into Eq. 2.21, we have

$\Omega_{L_{n}^{*}}\left(p_{n}, p_{i}\right)=n-i$

$\Omega_{L_{n}^{*}}\left(p_{n}, q_{i}\right)=n-i$

$$
+\frac{\left(1-\alpha^{n-i}\right)\left(2-2 \alpha^{n+i}-\alpha^{n+i-1}-\alpha^{n-i+1}+\alpha^{2 i-1}+\alpha\right)}{4 \sqrt{2}\left(1-\alpha^{2 n}\right)},
$$

$$
+\frac{\left(1+\alpha^{n-i}\right)\left(2+2 \alpha^{n+i}+\alpha^{n+i-1}+\alpha^{n-i+1}+\alpha^{2 i-1}+\alpha\right)}{4 \sqrt{2}\left(1-\alpha^{2 n}\right)},
$$

$\Omega_{L_{n}^{*}}\left(p_{i}, q_{i}\right)=\frac{1+\alpha^{2 i-1}+\alpha^{2 n-2 i+1}+\alpha^{2 n}}{\sqrt{2}\left(1-\alpha^{2 n}\right)}$.

Finally, we compute $\Omega_{L_{n}^{*}}\left(p_{i}, p_{j}\right)$ and $\Omega_{L_{n}^{*}}\left(q_{i}, p_{j}\right)(n>i \geq j \geq 1)$. To this end, we consider $L_{n}^{*}$ as the union of two graphs: the upper part and the lower part with respect to $p_{i}$ and $q_{i}$, as illustrated in Figure 4. Note the lower part is the graph $L_{i}^{*}$, and the upper part is the graph $L_{n-i}^{*}$. Next, we make circuit reduction to $L_{n-i}^{*}$ so that it is reduced to an edge $p_{i+1} q_{i+1}$ with resistance $z_{n-i}$. Then, we reduce $L_{i}^{*}$ to a $Y$-shaped graph with end vertices $p_{i}, q_{i}$, and $p_{j}$, and resistances $D, E$, and $F$ along its edges. These reductions are illustrated in Figure 4. Then, we have

$D+E=\Omega_{L_{i}^{*}}\left(p_{i}, p_{j}\right), D+F=\Omega_{L_{i}^{*}}\left(p_{i}, q_{i}\right)=z_{i}, E+F=\Omega_{L_{i}^{*}}\left(q_{i}, p_{j}\right)$.

It follows that

$$
\begin{aligned}
& D=\frac{\Omega_{L_{i}^{*}}\left(p_{i}, p_{j}\right)+z_{i}-\Omega_{L_{i}^{*}}\left(q_{i}, p_{j}\right)}{2}, \\
& E=\frac{\Omega_{L_{i}^{*}}\left(p_{i}, p_{j}\right)-z_{i}+\Omega_{L_{i}^{*}}\left(q_{i}, p_{j}\right)}{2}, \\
& F=\frac{-\Omega_{L_{i}^{*}}\left(p_{i}, p_{j}\right)+z_{i}+\Omega_{L_{i}^{*}}\left(q_{i}, p_{j}\right)}{2} .
\end{aligned}
$$

On the other hand, by the series and parallel connection rules, we have

$$
\begin{aligned}
& \Omega_{L_{n}^{*}}\left(p_{i}, p_{j}\right)=\frac{D\left(z_{n-i}+F+4\right)}{z_{n-i}+z_{i}+4}+E, \\
& \Omega_{L_{n}^{*}}\left(q_{i}, p_{j}\right)=\frac{F\left(z_{n-i}+D+4\right)}{z_{n-i}+z_{i}+4}+E .
\end{aligned}
$$

By Eqs. (2.8), Eqs 2.22-2.25, and doing some algebra using Mathematica [30], we obtain

$$
\begin{aligned}
& \Omega_{L_{n}^{*}}\left(p_{i}, p_{j}\right)=i-j \\
& \quad+\frac{\left(1-\alpha^{i-j}\right)\left(2-\alpha^{i+j-1}+\alpha^{2 j-1}+\alpha^{2 n-2 i+1}\left(1-\alpha^{i-j}-2 \alpha^{i+j-1}\right)\right)}{4 \sqrt{2}\left(1-\alpha^{2 n}\right)},
\end{aligned}
$$

$\Omega_{L_{n}^{*}}\left(q_{i}, p_{j}\right)=i-j$

$$
+\frac{\left(1+\alpha^{i-j}\right)\left(2+\alpha^{i+j-1}+\alpha^{2 j-1}+\alpha^{2 n-2 i+1}\left(1+\alpha^{i-j}+2 \alpha^{i+j-1}\right)\right)}{4 \sqrt{2}\left(1-\alpha^{2 n}\right)} .
$$

It is easily verified that Eq. 2.27 is valid for $i=j$.

Step 2. Computation of resistance distances between $p, q \in V_{2}$ and between $p \in V_{1}$ and $q \in V_{2}$.

First, we compute $\Omega_{L_{n}}\left(s_{i}, p_{i}\right)$ and $\Omega_{L_{n}}\left(s_{i}, p_{i+1}\right)$. Applying Lemma 2.1 to pairs of vertices $\left\{s_{i}, p_{i}\right\}$ and $\left\{s_{i}, p_{i+1}\right\}$, we obtain

$$
\begin{aligned}
& 2 \Omega_{L_{n}}\left(s_{i}, p_{i}\right)+\Omega_{L_{n}}\left(p_{i}, s_{i}\right)-\Omega_{L_{n}}\left(p_{i}, p_{i}\right)+\Omega_{L_{n}}\left(p_{i+1}, s_{i}\right) \\
& \quad-\Omega_{L_{n}}\left(p_{i+1}, p_{i}\right)=2, \\
& 2 \Omega_{L_{n}}\left(s_{i}, p_{i+1}\right)+\Omega_{L_{n}}\left(p_{i}, s_{i}\right)-\Omega_{L_{n}}\left(p_{i}, p_{i+1}\right)+\Omega_{L_{n}}\left(p_{i+1}, s_{i}\right) \\
& \quad-\Omega_{L_{n}}\left(p_{i+1}, p_{i+1}\right)=2 .
\end{aligned}
$$

Multiplying Eq. 2.28 by 3 and then minus Eq. 2.29, we get

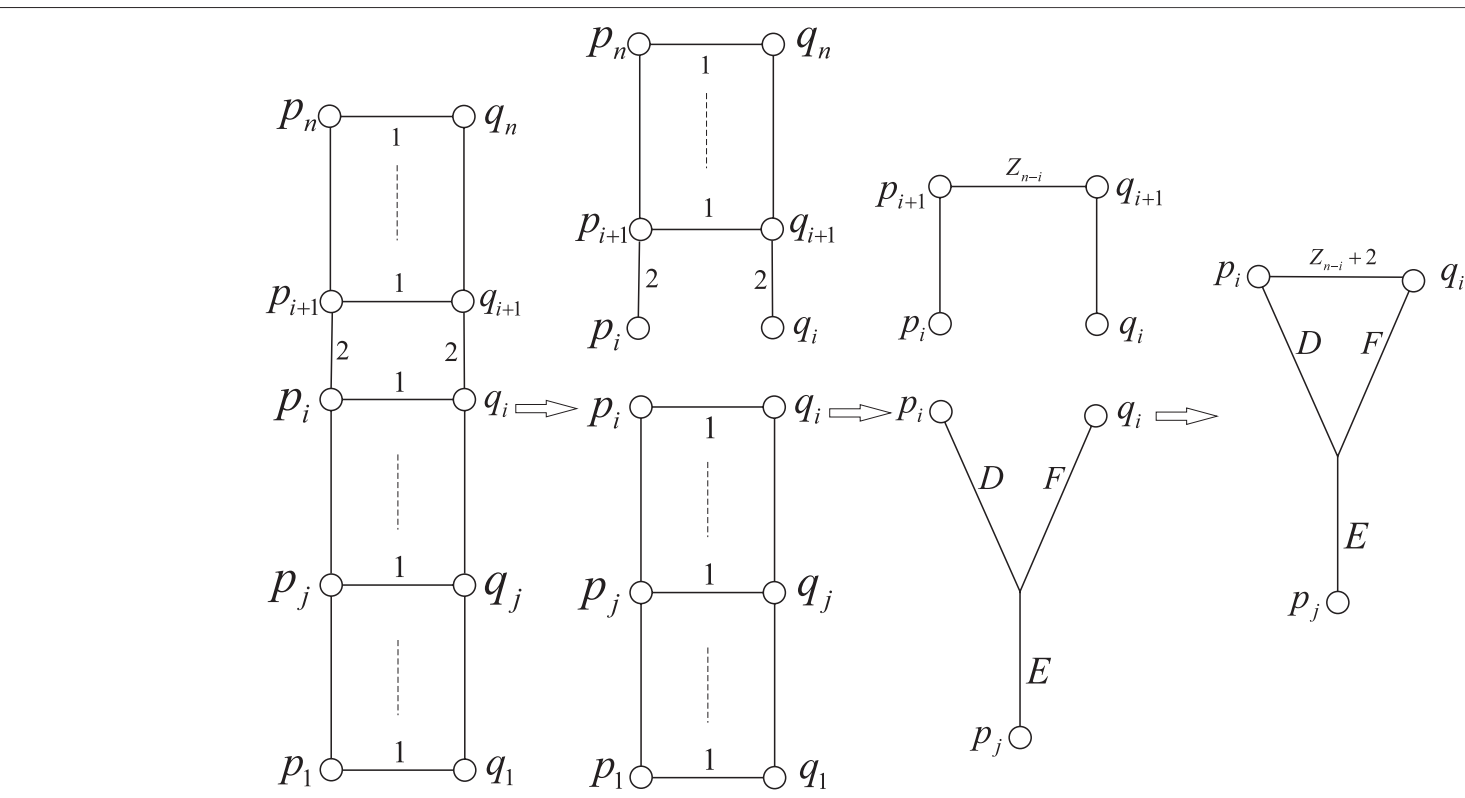

FIGURE $4 \mid L_{n}^{*}$ and circuit reductions to find $\Omega_{L_{n}^{*}}\left(p_{i}, p_{j}\right)$ and $\Omega_{L_{n}^{*}}\left(q_{i}, p_{j}\right)$. 


$$
\Omega_{L_{n}}\left(s_{i}, p_{i}\right)=\frac{1}{8}\left(4+2 \Omega_{L_{n}}\left(p_{i}, p_{i+1}\right)\right) .
$$

Then, substituting the value of $\Omega_{L_{n}}\left(p_{i}, p_{i+1}\right)$ as obtained in Step 1 into Eq. 2.30, we could obtain

$$
\begin{aligned}
& \Omega_{L_{n}}\left(p_{i+1}, p_{i}\right)=1 \\
& \quad+\frac{(1-\alpha)\left(2-\alpha^{2 i}+\alpha^{2 i-1}+\alpha^{2 n-2 i-1}\left(1-\alpha-2 \alpha^{2 i}\right)\right)}{4 \sqrt{2}\left(1-\alpha^{2 n}\right)} .
\end{aligned}
$$

Substituting Eq. 2.31 into Eq. 2.30, we have

$\Omega_{L_{n}}\left(s_{i}, p_{i}\right)=\frac{3}{4}+\frac{(1-\alpha)\left(2-\alpha^{2 i}+\alpha^{2 i-1}+\alpha^{2 n-2 i-1}\left(1-\alpha-2 \alpha^{2 i}\right)\right)}{16 \sqrt{2}\left(1-\alpha^{2 n}\right)}$.

In the same way, we could obtain that

$$
\begin{aligned}
\Omega_{L_{n}}\left(s_{i}, p_{i+1}\right)= & \frac{3}{4} \\
& +\frac{(1-\alpha)\left(2-\alpha^{2 i}+\alpha^{2 i-1}+\alpha^{2 n-2 i-1}\left(1-\alpha-2 \alpha^{2 i}\right)\right)}{16 \sqrt{2}\left(1-\alpha^{2 n}\right)} .
\end{aligned}
$$

Second, we calculate the resistance distance between $s_{i}$ and $p_{j}$. Again, applying Lemma 2.1 to $\left\{s_{i}, p_{j}\right\}$, we obtain

$$
\begin{aligned}
& 2 \Omega_{L_{n}}\left(s_{i}, p_{j}\right)+\Omega_{L_{n}}\left(p_{i}, s_{i}\right)-\Omega_{L_{n}}\left(p_{i}, p_{j}\right)+\Omega_{L_{n}}\left(p_{i+1}, s_{i}\right) \\
& \quad-\Omega_{L_{n}}\left(p_{i+1}, p_{j}\right)=2 .
\end{aligned}
$$

By Eqs 2.32, 2.33, it follows that

$$
\begin{aligned}
& \Omega_{L_{n}}\left(p_{i}, s_{i}\right)+\Omega_{L_{n}}\left(p_{i+1}, s_{i}\right)=\frac{3}{2} \\
& \quad+\frac{(1-\alpha)\left(2-\alpha^{2 i}+\alpha^{2 i-1}+\alpha^{2 n-2 i-1}\left(1-\alpha-2 \alpha^{2 i}\right)\right)}{8 \sqrt{2}\left(1-\alpha^{2 n}\right)} .
\end{aligned}
$$

For the sake of simplicity, we define

$$
\begin{aligned}
f(x, y)= & \left(1-\alpha^{x-y}\right)\left(2-\alpha^{x+y-1}+\alpha^{2 y-1}+\alpha^{2 n-2 x+1}\left(1-\alpha^{x-y}\right.\right. \\
& \left.\left.-2 \alpha^{x+y-1}\right)\right) .
\end{aligned}
$$

Then, Eq. 2.35 can be rewritten as

$$
\Omega_{L_{n}}\left(p_{i}, s_{i}\right)+\Omega_{L_{n}}\left(p_{i+1}, s_{i}\right)=\frac{3}{2}+\frac{f(i+1, i)}{8 \sqrt{2}\left(1-\alpha^{2 n}\right)} .
$$

On the other hand, by Eq. 2.26, we have

$$
\Omega_{L_{n}}\left(p_{i}, p_{j}\right)+\Omega_{L_{n}}\left(p_{i+1}, p_{j}\right)=2 i-2 j+1+\frac{f(i, j)+f(i+1, j)}{4 \sqrt{2}\left(1-\alpha^{2 n}\right)} .
$$

Substituting Eqs. 2.37, 2.38 into Eq. 2.34, we draw the conclusion that

$$
\Omega_{L_{n}}\left(s_{i}, p_{j}\right)=i-j+\frac{3}{4}-\frac{f(i+1, i)}{16 \sqrt{2}\left(1-\alpha^{2 n}\right)}+\frac{f(i, j)+f(i+1, j)}{8 \sqrt{2}\left(1-\alpha^{2 n}\right)} .
$$

Third, we calculate the resistance distance between $s_{j}$ and $q_{i}$. Apply Lemma 2.1 to $\left\{s_{j}, q_{i}\right\}$ to obtain

$$
\begin{aligned}
& 2 \Omega_{L_{n}}\left(s_{j}, q_{i}\right)+\Omega_{L_{n}}\left(p_{j}, s_{j}\right)-\Omega_{L_{n}}\left(p_{j}, q_{i}\right)+\Omega_{L_{n}}\left(p_{j+1}, s_{j}\right) \\
& \quad-\Omega_{L_{n}}\left(p_{j+1}, q_{i}\right)=2 .
\end{aligned}
$$

By Eq. 2.37, we have

$$
\Omega_{L_{n}}\left(p_{j}, s_{j}\right)+\Omega_{L_{n}}\left(p_{j+1}, s_{j}\right)=\frac{3}{2}+\frac{f(j+1, j)}{8 \sqrt{2}\left(1-\alpha^{2 n}\right)} .
$$

For simplicity, we define

$$
\begin{aligned}
g(x, y)= & \left(1+\alpha^{x-y}\right)\left(2+\alpha^{x+y-1}+\alpha^{2 y-1}+\alpha^{2 n-2 x+1}\left(1+\alpha^{x-y}\right.\right. \\
& \left.\left.+2 \alpha^{x+y-1}\right)\right)
\end{aligned}
$$

On the other hand, by Eq. 2.27, we have

$$
\Omega_{L_{n}}\left(q_{i}, p_{j}\right)+\Omega_{L_{n}}\left(q_{i}, p_{j+1}\right)=2 i-2 j-1+\frac{g(i, j)+g(i, j+1)}{4 \sqrt{2}\left(1-\alpha^{2 n}\right)} .
$$

Substituting Eqs. 2.41-2.43 into Eq. 2.40, we get

$$
\Omega_{L_{n}}\left(s_{j}, q_{i}\right)=i-j-\frac{1}{4}+\frac{f(i+1, i)}{16 \sqrt{2}\left(1-\alpha^{2 n}\right)}+\frac{g(i, j)+g(i, j+1)}{8 \sqrt{2}\left(1-\alpha^{2 n}\right)} .
$$

Fourth, we calculate the resistance distance between $s_{i}$ and $s_{j}$. Applying Lemma 2.1 to $\left\{s_{i}, s_{j}\right\}$, we have

$$
\begin{aligned}
& 2 \Omega_{L_{n}}\left(s_{i}, s_{j}\right)+\Omega_{L_{n}}\left(p_{i}, s_{i}\right)-\Omega_{L_{n}}\left(p_{i}, s_{j}\right)+\Omega_{L_{n}}\left(p_{i+1}, s_{i}\right) \\
& \quad-\Omega_{L_{n}}\left(p_{i+1}, s_{j}\right)=2 .
\end{aligned}
$$

As $\Omega_{L_{n}}\left(p_{i}, s_{i}\right), \Omega_{L_{n}}\left(p_{i}, s_{j}\right), \Omega_{L_{n}}\left(p_{i+1}, s_{i}\right)$, and $\Omega_{L_{n}}\left(p_{i+1}, s_{j}\right)$ have been given by Eq. 2.39, simple calculation leads to

$$
\begin{aligned}
& \Omega_{L_{n}}\left(s_{i}, s_{j}\right)=\frac{1}{2}-i+j \\
& -\frac{f(i+1, i)+f(j+1, j)+f(j, i)+f(j+1, i)+f(j, i+1)+f(j+1, i+1)}{16 \sqrt{2}\left(1-\alpha^{2 n}\right)} .
\end{aligned}
$$

Fifth and finally, we calculate the resistance between $s_{i}$ and $t_{j}$. Applying Lemma 2.1 to $\left\{s_{i}, t_{j}\right\}$, we have

$$
\begin{aligned}
& 2 \Omega_{L_{n}}\left(s_{i}, t_{j}\right)+\Omega_{L_{n}}\left(p_{i}, s_{i}\right)-\Omega_{L_{n}}\left(p_{i}, t_{j}\right)+\Omega_{L_{n}}\left(p_{i+1}, s_{i}\right) \\
& \quad-\Omega_{L_{n}}\left(p_{i+1}, t_{j}\right)=2
\end{aligned}
$$

Note by the symmetry of $L_{n}$ that we have $\Omega_{L_{n}}\left(p_{i}, t_{j}\right)=$ $\Omega_{L_{n}}\left(q_{i}, s_{j}\right)$ and $\Omega_{L_{n}}\left(p_{i+1}, t_{j}\right)=\Omega_{L_{n}}\left(q_{i+1}, s_{j}\right)$. Using the results obtained in Eqs. 2.39-2.44, simple algebraic calculation yields 


$$
\begin{aligned}
\Omega_{L_{n}}\left(s_{i}, t_{j}\right)=\frac{1}{2}+i-j \\
+\frac{g(i, j)+g(i, j+1)+g(i+1, j)+g(i+1, j+1)-f(i+1, i)}{16 \sqrt{2}\left(1-\alpha^{2 n}\right)} \\
\quad-\frac{f(i+1, i)+f(i+2, i+1)}{32 \sqrt{2}\left(1-\alpha^{2 n}\right)} .
\end{aligned}
$$

\section{CONCLUSION}

The computation of resistance distances is a classical problem in electrical circuit theory, which has attracted much attention. It is of special interest to investigate resistance distances in plane networks. Along this line, we have considered the linear polyacene network, with exact expression for resistance distances in this network being given. It is a primary attempt for the computation of resistance distances in plane hexagonal lattice. Resistance distances in more and more plane hexagonal lattices are greatly anticipated.

\section{REFERENCES}

1. Klein DJ, Randić M. Resistance distance. J Math Chem (1993) 12:81-95. doi:10. 1007/BF01164627

2. Shapiro LW. An electrical lemma. Math Mag (1987) 60:36-8.

3. Nash Williams JAC, St. Random walks and electric currents in networks. Proc. Cambridge Phil Soc (1959) 55:181-94. 10.1017/S0305004100033879

4. Doyle PG, Snell JL. Random walks and electric networks. Washington, DC: The Mathematical Association of America (1984) 118 p.

5. Lukovits I, Nikolić S, Trinajstić N. Resistance distance in regular graphs. Int J Quant Chem (1999) 71:217-25. 10.1002/(SICI)1097-461X(1999)71:3<217:: AID-QUA1>3.0.CO;2-C

6. Fowler PW. Resistance distances in fullerene graphs. Croat Chem Acta (2002) 75:401-8.

7. Zhang H, Yang Y. Resistance distance and Kirchhoff index in circulant graphs. Int J Quant Chem (2007) 107:330-9. 10.1002/qua.21068

8. Palacios JL. Closed-form formulas for Kirchhoff index. Int J Quant Chem (2001) 81:135-40. 10.1002/1097-461X(2001)81:2\%3C135::AID-QUA4\%3E3.0.CO;2-G

9. Jafarizadeh MA, Sufiani R, Jafarizadeh S. Recursive calculation of effective resistances in distance-regular networks based on Bose-Mesner algebra and Christoffel-Darboux identity. J Math Phys (2009) 50:023302. doi:10.1063/1. 3077145

10. Jafarizadeh S, Sufiani R, Jafarizadeh MA. Evaluation of effective resistances in pseudo-distance-regular resistor networks. J Stat Phys (2010) 139:177-99. doi:10.1007/s10955-009-9909-8

11. Bapat RB, Gupta S. Resistance distance in wheels and fans. Indian J Pure Appl Math (2010) 41:1-13. doi:10.1007/s13226-010-0004-2

12. Gao X, Luo Y, Liu W. Resistance distances and the Kirchhoff index in Cayley graphs. Discrete Appl Math (2011) 159:2050-7. doi:10.1016/j.dam.2011.06.027

13. Chair N. Exact two-point resistance, and the simple random walk on the complete graph minus N edges. Ann Phys (2012) 327:3116-29. doi:10.1016/j.aop.2012.09.002

14. Tan Z, Essam JW, Wu FY. Two-point resistance of a resistor network embedded on a globe. Phys Rev E Stat Nonlinear Soft Matter Phys (2014) 90:012130. doi:10.1103/PhysRevE.90.012130

15. Chair N, Ali Dannoun EM. Two-point resistance of the Möbius ladder. Phys Scripta (2015) 90:035206. doi:10.1088/0031-8949/90/3/035206

16. Tan Z. Theory on resistance of $m \times n$ cobweb network and its application. Int J Circ Theor Appl (2015) 43:1687-702. doi:10.1002/cta.2035

17. Gervacio SV. Resistance distance in complete $n$-partite graphs. Discrete Appl Math (2016) 203:53-61. doi:10.1016/j.dam.2015.09.017

\section{DATA AVAILABILITY STATEMENT}

The original contributions presented in the study are included in the article/Supplementary Material; further inquiries can be directed to the corresponding author.

\section{AUTHOR CONTRIBUTIONS}

All authors listed have made a substantial, direct, and intellectual contribution to the work and approved it for publication.

\section{FUNDING}

This research was funded by the National Natural Science Foundation of China through grant number 116711347, and project ZR2019YQ02 by Shandong Provincial Natural Science Foundation.

\section{ACKNOWLEDGMENTS}

We would like to thank the anonymous reviewers for their useful comments.

18. Tan Z. Two-point resistance of an $m \times n$ resistor network with an arbitrary boundary and its application in RLC network. Chin Phys B (2016) 25:050504. doi:10.1088/1674-1056/25/5/050504

19. Cinkir Z. Effective resistances and Kirchhoff index of a ladder graph. J Math Chem (2016) 54:955-66. doi:10.1007/s10910-016-0597-8

20. Tan Z, Asad JH, Owaidat MQ. Resistance formulae of a multipurpose ?-step network and its application in LC network. Int J Circuit Theor Appl (2017) 45: 1942-57. doi:10.1002/cta.2366

21. Vaskouski M, Zadorozhnyuk A. Resistance distances in Cayley graphs on symmetric groups. Discrete Appl Math (2017) 227:121-35. doi:10.1016/j.dam.2017.04.044

22. Shangguan $Y$, Chen H. Two-point resistances in an Apollonian network. Phys Rev E (2017) 96:062140. doi:10.1103/PhysRevE.96.062140

23. Jiang Z, Yan W. Some two-point resistances of the Sierpinski gasket network. J Stat Phys (2018) 172:824-32. doi:10.1007/s10955-018-2067-0

24. Owaidat MQ, Asad JH, Tan Z. Resistance computation of generalized decorated square and simple cubic network lattices. Results Phys (2019) 12: 1621-7. doi:10.1016/j.rinp.2019.01.070

25. Shangguan $\mathrm{Y}$, Chen $\mathrm{H}$. Two-point resistances in a family of self-similar (x,y)flower networks. Physica A (2019) 523:382-91. doi:10.1016/j.physa.2019.02.008

26. Ye L, Yan W. Resistance between two vertices of almost complete bipartite graphs. Discrete Appl Math (2019) 257:299-305. doi:10.1016/j.dam.2018.08.030

27. Barrett W, Evans EJ, Francis AE. Resistance distance in straight linear 2-trees. Discrete Appl Math (2019) 258:13-34. doi:10.1016/j.dam.2018.10.043

28. Jiang Z, Yan W. Resistances between two nodes of a path network. Appl Math Comput (2019) 361:42-6. doi:10.1016/j.amc.2019.05.006

29. Chen H, Zhang F. Resistance distance local rules. J Math Chem (2008) 44: 405-17. 10.1007/s10910-007-9317-8

30. Wolfram Research, Inc. Mathematica. version 9.0. Champaign, IL: Wolfram research Inc. (2012).

Conflict of Interest: The authors declare that the research was conducted in the absence of any commercial or financial relationships that could be construed as a potential conflict of interest.

Copyright (c) 2021 Wang and Yang. This is an open-access article distributed under the terms of the Creative Commons Attribution License (CC BY). The use, distribution or reproduction in other forums is permitted, provided the original author(s) and the copyright owner(s) are credited and that the original publication in this journal is cited, in accordance with accepted academic practice. No use, distribution or reproduction is permitted which does not comply with these terms. 